\title{
Evaluation der Benutzungsmodalitäten in der Musiksammlung der WLB
}

Der Wunsch nach Verbesserung des Benutzungsund Serviceangebots im Bereich der Musiksammlung führte zu der Überlegung, eine Evaluation der Benutzungsmodalitäten durchzuführen. Eine weitere Motivation war, neben den sinkenden Benutzerzahlen, eine maßgebliche Veränderung in der Benutzung: die Integration des Präsenzbestands Musik in den Hauptlesesaal und die Ausgabe von Noten und Tonträgern im Sonderlesesaal im Jahr 2015. In diesem Kontext entstand das Bestreben, die Bedingungen für die Benutzung der Musiksammlung attraktiver zu gestalten.

Neue Situationen bieten allerdings auch neue Möglichkeiten und Chancen. Alte Strukturen und Organisationsformen können neu durchdacht und verändert werden. Zu diesem Zweck hat die Württembergische Landesbibliothek eine Projektarbeit angeboten und konnte zwei Studierende im Rahmen ihres Praxissemesters dafür gewinnen: Hannes Britz (HdM Stuttgart) und Julia Walz (TH Köln). Grundlage des Projekts war zunächst eine Bestandsaufnahme des Ist-Zustandes. Dabei sollten die verschiedenen Facetten des Medienangebots und der Benutzungsmodalitäten eruiert werden. Ihre Aufgabe war es, in einem ersten Schritt die intern geführten Statistiken der Musiksammlung auszuwerten. In einem zweiten Schritt sollte ein Vergleich ausgewählter Musikbibliotheken unter verschiedenen Aspekten erfolgen. Schließlich beinhaltete der größte Teil des Projekts die Konzeption, Durchführung und Auswertung einer Benutzerumfrage. Alle drei Schritte führten am Ende zu einem Fazit, das neue Ideen zur Verbesserung der Benutzungsmodalitäten aufzeigt. ${ }^{1}$

In diesem Artikel sollen die wesentlichen Aspekte der Projektarbeit aufgezeigt werden. Den Anfang bildet eine Skizzierung des Ist-Zustandes (2016), der durch ausgewählte Statistiken über die letzten zehn Jahre detaillierter beschrieben wird. Es folgt die Darstellung und Bewertung der Ergebnisse der Benutzerumfrage. In einem abschließenden Fazit sollen die Erkenntnisse und die folgenden prakti- schen Konsequenzen aus der Projektarbeit für die Musiksammlung vorgestellt werden.

\section{Ist-Zustand (Ende 2016)}

Ende des Jahres 2016 stellte sich die Situation wie folgt dar:

- Im Laufe der letzten Jahre (besonders der letzten zehn Jahre) wurde ein kontinuierlicher Rückgang von Benutzern der Musiksammlung² festgestellt.

- Die Nutzung des Musikbestands veränderte sich bereits im Laufe der letzten (circa) zehn Jahre, vor allem aber seit Februar 2015 mit Abriss des ehemals vorhandenen Musiklesesaals. Konnte früher der Bestand vollständig im eigenen Musiklesesaal benutzt werden, erfolgt seither die Ausgabe der Noten im Sonderlesesaal (Öffnungszeiten: 10-17 Uhr), der Präsenzbestand ist jedoch im Hauptlesesaal (Öffnungszeiten: 8-20 Uhr) benutzbar.

- Noten und Tonträger sind grundsätzlich nicht nach Hause, sondern nur in den Sonderlesesaal entleihbar.

- Bis Anfang 2017 wurden insgesamt fünf Kataloge zur Benutzung der Musik-Bestände auf der Website angeführt, die eine gewisse Unübersichtlichkeit mit sich brachten und die Benutzung erschwerten. Durch die notwendige Recherche sowohl im digitalisierten Zettel- als auch im Online-Katalog kam es zu einer Verkomplizierung des Bestellvorganges.

- Bedingt durch die Einführung eines neuen Bibliothekssystems, konnten über drei Jahre hinweg die Neuzugänge der Noten nicht katalogisiert werden.

\section{Auswertung der Statistiken}

Die Auswertung der Statistiken der Jahre 2006 bis 2015/2016 mithilfe von grafischen Darstellungen soll die Entwicklung der erhobenen Zahlen dokumentieren. Untersucht wurden die nachfolgend ge-

1) Dieser Artikel basiert auf den Ergebnissen des intern vorliegenden Projektberichts. Die hier abgebildeten Diagramme wurden von Hannes Britz und Julia Walz erstellt.

2) Mit Musiksammlung sind in erster Linie der Notenbestand und auch die Musik-Tonträger gemeint, beides wird im Sonderlesesaal ausgegeben. Keine Berücksichtigung findet hier die musikwissenschaftliche Fachliteratur. 
nannten Statistiken, auf die im Folgenden teilweise eingegangen wird.

1) Nutzung der Musiksammlung im (Musik-) Lesesaal

2) mündliche und schriftliche Anfragen der Nutzer an das Musikpersonal

3) Musikdatenbankzugriffe

4) Zugriffe auf Musikzeitschriften durch die elektronische Zeitschriftendatenbank (EZB)

5) Fernleihe des Musikbestandes

6) Fachführungen Musik

7) Nutzung des Flügels und der Audiokabine

In den Jahren 2006 bis 2014 gingen die Benutzerzahlen konstant von anfangs über 3.600 pro Jahr (2006) auf weniger als die Hälfte zurück (ca. 1.500 im Jahr 2014). Ebenso hat die Bestellung von Noten aus dem Magazin deutlich abgenommen. Wurden im Jahr 2006 noch etwas mehr als 1.300 Musikdrucke im Jahr ausgehoben, waren es 2016 nur noch knapp 400.

Erwähnenswert ist die Statistik der aktiven Fernleihe der Musiksammlung. In den vergangenen Jahrzehnten hat man sich sehr restriktiv mit der Ausleihe von Musikalien an andere Bibliotheken verhalten. Nur sehr wenige Bestellungen konnten positiv bearbeitet werden. Im Jahr 2015 wurden die Ausleihbedingungen maßgeblich verändert. Auslöser hierfür war eine berechtigte Diskussion in der AG Fernleihe hinsichtlich der bis dahin nur selten gestatteten Ausleihe von Musikalien aus der WLB. Die Auswirkungen dieser Entscheidung machten sich bereits im Jahr 2016 mit deutlich höheren Ausleihzahlen bemerkbar: 2016 hat sich die Zahl der aktiven Fernleihen im Gegensatz zum Vorjahr bereits mehr als verdoppelt.

Benutzerführungen in der Musiksammlung werden in erster Linie für Studierende der Staatlichen Hochschule für Musik und Darstellende Kunst (HMDK) Stuttgart durchgeführt. Hier handelt es sich jedoch um verpflichtende Teilnahme im Rahmen bestimmter Kurse. Gelegentlich werden Führungen auf Wunsch einzelner Besuchergruppen veranstaltet.

Im Sonderlesesaal der Landesbibliothek befindet sich sowohl ein Flügelraum als auch eine Audioka- bine mit Abspielgeräten für Schallplatten, Tonkassetten und CDs. Beide Räume werden verhältnismäßig wenig genutzt. Außer diesen Abspielgeräten steht noch ein tragbarer CD-Spieler zur Verfügung, der gelegentlich benutzt wird. CDs werden jedoch eher mit dem eigenen Laptop abgespielt.

\section{Vergleich mit anderen (Musik-) Bibliotheken}

Um Neuerungen und Verbesserungen der eigenen Serviceangebote zu erreichen, ist es hilfreich, einen Blick auf andere ausgewählte (Musik-)Bibliotheken zu werfen. Für einen Vergleich wurden die folgenden Einrichtungen hinzugezogen: Die Stadtbibliothek Stuttgart (Ebene Musik) und die Bibliothek der Staatlichen Hochschule für Musik und Darstellende Kunst (HDMK) wurden aufgrund der lokalen Nähe miteinbezogen. Die Landesbibliothek Speyer und die Badische Landesbibliothek Karlsruhe wurden als zwei weitere Regionalbibliotheken mit ähnlichem Benutzerprofil ausgesucht. Die Schwerpunkte dieses Vergleichs lagen auf der Entleihbarkeit des Bestandes (vor allem des Pflichtbestandes), Zusammensetzung und Auswahl des Lesesaalbestandes, der Öffentlichkeitsarbeit und der Art der Nutzergruppen.

\section{Benutzerumfrage in der WLB}

Die Nutzerbefragung stellte den wesentlichen Teil des Projekts dar. Sie sollte primär Erkenntnisse über die Art der Benutzer, ihren Umgang mit den vorhandenen Informationsressourcen (z. B. Kataloge, Website) sowie ihre Erwartungen und Wünsche an die WLB hinsichtlich Bestand und Dienstleistungsangebot erbringen.

Die Umfrage fand vom 12. Dezember 2016 bis 5. Januar 2017 statt und wurde zeitgleich in der WLB auf der Infostehle und mit Plakaten (inkl. QRCode) in den Lesesälen beworben. Außerdem wurden Plakate in der Bibliothek der Musikhochschule aufgehängt. Darüber hinaus nutzte man unterschiedliche Online-Kanäle: Facebook, verschiedene Bereiche der Website und der WLB-Newsletter. 
Die Umfrage beinhaltete die folgenden Fragen: ${ }^{3}$

Wie alt sind Sie?

Aus welchem Grund nutzen Sie den WLB-Musikbestand? (Mehrfachauswahl möglich)

Den Wegfall des Musiklesesaals empfinde ich als ...

Wie beurteilen Sie die Tatsache, dass die Bestände des früheren Musiklesesaals nun im Hauptlesesaal und die zu bestellenden Noten jetzt im Sonderlesesaal zu benutzen sind?

Wie beurteilen Sie die im Vergleich zum Hauptlesesaal kürzeren Öffnungszeiten des Sonderlesesaals?

Bitte beurteilen Sie das Medienangebot im Hauptlesesaal:

Bibliographien und Kataloge

Lexika und weitere Nachschlagewerke

Gesamt- und Denkmälerausgaben

Literatur zur Musikgeschichte Baden-Württembergs

Wie wichtig wäre Ihnen eine Entleihbarkeit nach Hause von Noten und Musik-CDs?

Welche Onlineangebote nutzen Sie?

Auf der Webseite wird deutlich erklärt, in welchem Katalog welche Teilbestände nachgewiesen sind...

Wie beurteilen Sie die Anwendungsfreundlichkeit der Kataloge? (WLB-Katalog, DigiKat Musik, DigiKat (WLB), DigiSyk Musik, Musik-OPAC des SWB, Systematischer Katalog Musik)

Wenn ich Fragen zum Musikbestand habe, weiß ich, an wen ich mich wenden kann ...

Wie beurteilen Sie die Erreichbarkeit der Musikbibliothekarinnen der WLB? (Per Mail, Per Telefon, im Gespräch vor Ort)

Die Qualität der Auskunft des Sonderlesesaalpersonals ist ...

Die Qualität der Auskunft der Musikbibliothekarinnen ist ...

Wussten Sie, dass die Musiksammlung der WLB ..

die Musikliteratur (auch Noten und Tonträger) der württembergischen Verlage besitzt?

einen Steinway-Flügel zum Anspielen der Noten anbietet?

die Aufführungsmaterialien der Staatstheater Stuttgart sammelt?

Musikfachführungen anbietet?

ein umfassendes musikspezifisches Datenbankangebot lizenziert hat? im Sonderlesesaal einen Buchscanner zur Verfügung stellt?

Haben Sie Verbesserungsvorschläge, Wünsche oder Kritik? Dann dürfen Sie uns diese gerne mitteilen.

Sehr schnell stellte sich heraus, dass die Nutzer in erster Linie Gebrauch von der Onlineversion der Umfrage machten. Online nahmen insgesamt 149 Nutzer teil. Davon führten 80 die Umfrage vollständig durch, 69 weitere beantworteten mindestens eine Frage nicht. Der Link zur Umfrage wurde 430 mal angeklickt. Dabei erfolgten die meisten Abbrüche auf der Startseite des Fragebogens. Begründet werden können die Abbrüche möglicherweise damit, dass vielfach eine allgemeine Nutzerumfrage erwartet wurde. Die meisten Onlinezugriffe erfolgten in den ersten beiden Wochen. Die Papierversion wurde hingegen wider Erwarten lediglich achtmal ausgefüllt.

\section{Ergebnisse der Umfrage Alter und Hintergrund}

Den zahlenmäßig größten Teil der Benutzer macht die Gruppe der 20- bis 30-Jährigen aus (42 von 125 Teilnehmern), gefolgt von den 30- bis 40-Jährigen (20 von 125). Bei der hohen Anzahl der 20- bis 30-Jährigen handelt es sich wohl in erster Linie um Nutzer der Fachliteratur zur Musikwissenschaft. ${ }^{4}$

Bei der Frage nach dem Nutzungsgrund (insgesamt 130 Antworten auf diese Frage) werden zunächst die privaten Zwecke genannt (siehe Abb. 1). An zweiter Stelle folgen als Gründe Studium und Forschung. Da bei dieser Frage eine Mehrfachnennung möglich war, ist es wahrscheinlich, dass hier in Abgrenzung zu den rein beruflichen Zwecken der private Zweck zusätzlich gewählt wurde.

\section{Aus welchem Grund nutzen Sie den WLB-Musikbestand? (Mehrfachnennung war möglich)}

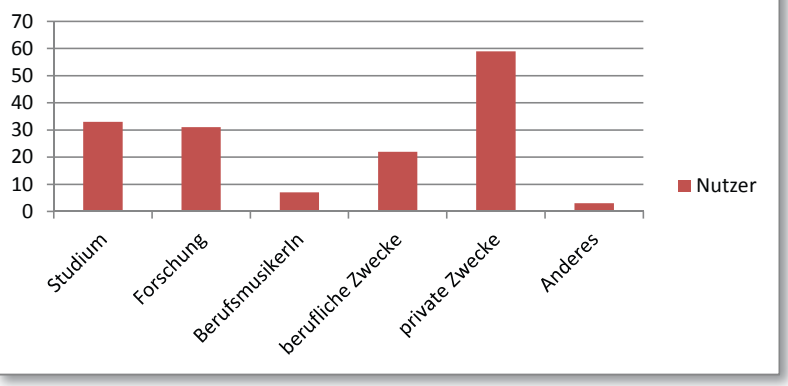

Abb. 1

Ergänzend zu der Frage nach dem Hintergrund der Benutzer, die in die WLB für Recherchen zur Musik kommen, wurde in der benachbarten Bibliothek der Staatlichen Hochschule für Musik und Darstellende Kunst eine kleine mündliche Umfrage durchgeführt. Ziel war es, herauszufinden, inwieweit die Studierenden Kenntnis von der Musiksammlung der WLB haben und ob sie den Bestand (und wenn ja wofür) nutzen. Zwar nicht alle, aber doch die Mehrheit von 50 der 60 Befragten wussten von der Musiksammlung. Allerdings gaben nur 28 an, den Bestand auch zu nutzen, hierbei jedoch ausschließlich die musikwissenschaftliche Fachliteratur, nicht aber die Noten. Begründet wurde die Nicht-Nutzung ${ }^{5}$ damit, dass der Notenbestand in der Hochschulbibliothek ausreichend sei.

3) Die Fragestellungen wurden von Herrn Britz und Frau Walz entwickelt. Abgedruckt sind hier aus Platzgründen lediglich die Fragen (ohne die Antwortmöglichkeiten).

4) $\mathrm{Vgl}$. unten die Ergebnisse der Umfrage in der Bibliothek der HMDK.

5) Zu den Nicht-Nutzern wurden auch diejenigen gezählt, die angaben, nur im Rahmen der Pflichtveranstaltung im ersten Semester in der WLB gewesen zu sein. 


\section{Medienangebot im Hauptlesesaal}

Mit dem Medienangebot im Hauptlesesaal waren die meisten Benutzer zufrieden, wenngleich die hohe Anzahl derjenigen, die „keine Angabe" gewählt hatten, auffällt. ${ }^{6}$ Grund hierfür ist möglicherweise die allgemein zu beobachtende Tatsache, dass verhältnismäßig wenige den Präsenzbestand Musik überhaupt nutzen. Bedarf an einem vergröBerten Angebot besteht vor allem im Bereich der Lexika und Nachschlagewerke und auch im Bereich der Literatur zur Musikgeschichte Baden-Württembergs. Der Mehrbedarf im Bereich Lexika liegt möglicherweise darin begründet, dass hier derzeit nur größere Nachschlagewerke stehen. Kleinere Lexika finden sich aber darüber hinaus im weiteren thematisch aufgestellten Bestand. Hinzuweisen ist an dieser Stelle auf die geplante Umsystematisierung des Lesesaalbestands auf die RVK (Regensburger Verbundklassifikation), die eine völlig neue Aufstellung des Bestands zur Folge hat.

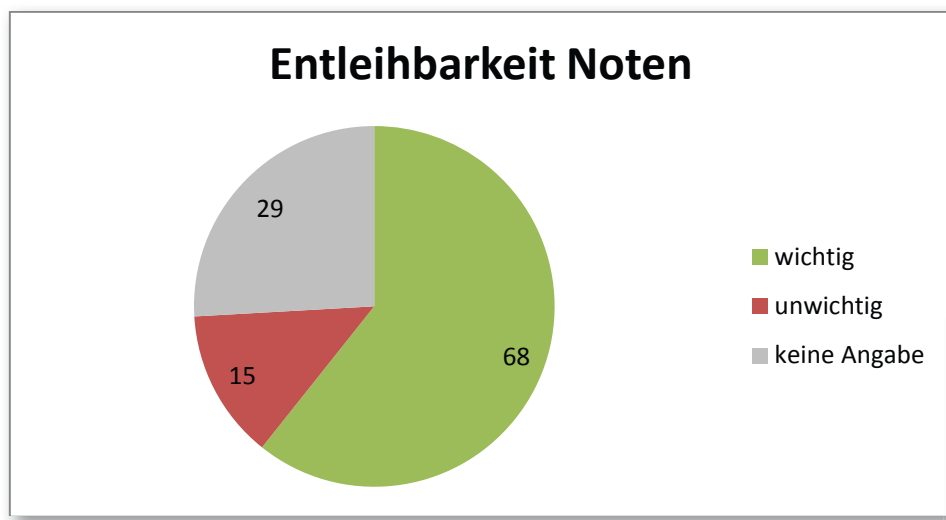

Abb. 2

\section{Entleihbarkeit der Medien}

Die beiden Abbildungen zeigen, dass erwartungsgemäß von Nutzerseite aus ein größerer Wunsch nach der (Außer-Haus-)Ausleihe vor allem von Noten besteht. Bei den Tonträgern ist lediglich die Hälfte an der Ausleihe interessiert, die andere Hälfte macht entweder keine Angabe oder hält die Ausleihe für unwichtig. Der mangelnde Wunsch nach Ausleihe der Tonträger lässt sich wahrscheinlich dadurch erklären, dass Musik-Tonträger immer mehr an Bedeutung verlieren und stattdessen Streamingdienste zum Anhören von Musik verwendet werden.

\section{Entleihbarkeit Musik-CDs}

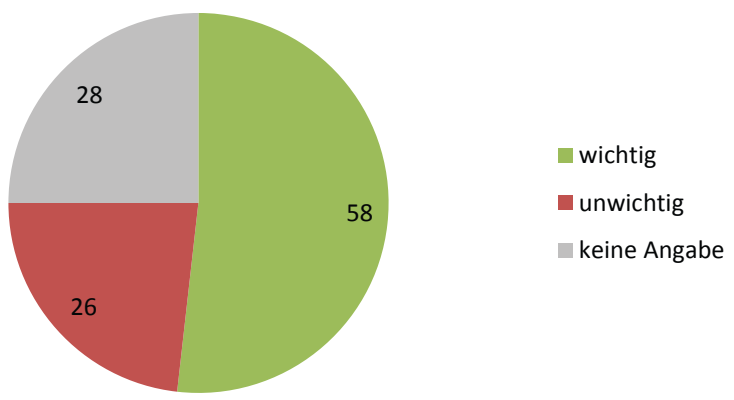

Abb. 3

\section{Anwendungsfreundlichkeit der Kataloge}

Die Anwendungsfreundlichkeit der Kataloge wird im Allgemeinen positiv beurteilt. Auffällig ist jedoch die hohe Zahl derjenigen, die angeben, die Kataloge noch nie genutzt zu haben. Dass der DigiSykMusik am wenigsten genutzt wird, erstaunt nicht: Schließlich ist der Katalog inhaltlich auf einem Stand von Anfang der 1990er Jahre und damit veraltet. Die weitgehende Nicht-Nutzung der anderen Kataloge (ausgenommen der allgemeine WLB-Katalog) liegt möglicherweise darin, dass die Recherche im DigiKat Musik eine völlig andere als im OPAC und deutlich anspruchsvoller ist.

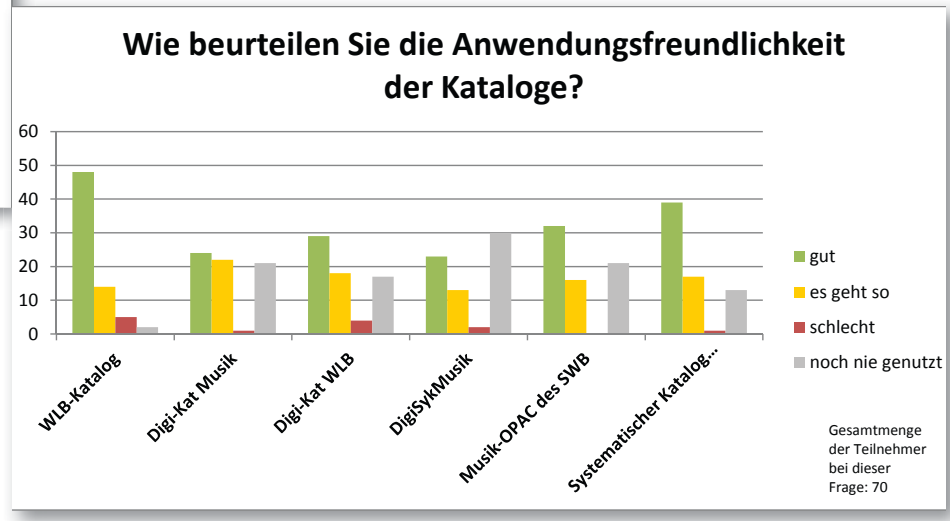

Abb. 4

\section{Nutzung weiterer Online-Angebote}

Zu den weiteren Online-Angeboten - neben den über die Website zugänglichen Katalogen - zählen die Musikdatenbanken, die Digitalen Sammlungen (vor allem Musikhandschriften) und die Website der Musiksammlung. Diese Angebote werden verhältnismäßig wenig genutzt. Am häufigsten werden mit 39\% (bei 102 Teilnehmern) die Datenbanken (für die Musikwissenschaft) in Anspruch genom- 
men, gefolgt von den Digitalen Sammlungen mit $32 \%$. Nur 29\% nutzen die Musik-Website und immerhin 25\% nutzen die weiteren Online-Angebote überhaupt nicht.

\section{Erklärung der Kataloge}

Bei 79 Antworten auf die Frage, ob auf der Website deutlich erklärt wird, in welchem Katalog welche Teilbestände nachgewiesen sind, machen 32 keine Angabe, 27 antworten mit "ja" und 20 finden die Erklärungen unzureichend. Grund hierfür mag eine gewisse Überforderung mit der bisherigen Vielzahl der genannten Kataloge und dem Umgang mit dem digitalisierten Zettelkatalog sein, der den heutigen Studierenden in der Regel fremd erscheint.

\section{Fragen zum Musikbestand}

\section{Bedingt durch den nicht mehr vorhandenen Mu-} siklesesaal und neue Wege zum Musikbestand war es wichtig zu erfragen, ob klar ist, wohin man sich mit Fragen zum Musikbestand wenden kann. Erfreulich ist, dass dies mehr als 50\% der Teilnehmer bekannt ist (46 von 80). Dennoch liegt der Anteil derer, die nicht wissen, an wen sie sich wenden können, bei fast einem Drittel (26 von 80). Möglicherweise schließen diese Antworten aber auch diejenigen ein, die noch nie einen Grund hatten, sich mit dieser Frage zu befassen.

\section{Erreichbarkeit}

Die Erreichbarkeit der Musikbibliothekarinnen (per E-Mail, per Telefon und im Gespräch vor Ort) wird überwiegend als gut empfunden. Auffällig bleibt aber die hohe Anzahl derjenigen, die noch gar keinen Kontakt gesucht haben. Bei insgesamt 79 Teilnehmern an dieser Frage haben 27 noch keinen Kontakt im Gespräch vor Ort gehabt, per Telefon und E-Mail waren es jeweils 50. Festzuhalten bleibt jedoch, dass der größte Teil der Kunden das Gespräch vor Ort sucht.

\section{Auskunftsqualität bei Sonderlesesaalpersonal und Musikbibliothekarinnen}

Nach Fragen zur Kenntnis über den richtigen Ansprechpartner und dessen Erreichbarkeit sollte in einem nächsten Schritt die Auskunftsqualität sowohl bei dem Sonderlesesaalpersonal als auch bei den Musikbibliothekarinnen untersucht werden. Die Bewertung fällt in beiden Bereichen überwiegend positiv aus. Dennoch antworten in allen drei Bereichen (Vollständigkeit der Auskunft, Dauer bis Erhalt der Auskunft, fachliche Qualität der Auskunft) auffallend viele Benutzer mit „weiß nicht" (z.B. stimmen insgesamt 65 Personen von 74 Teilnehmern im Hinblick auf das Sonderlesesaalpersonal und 73 Teilnehmern im Hinblick auf die Musikbibliothekarinnen für "weiß nicht”).

Die Klärung dieser Sachverhalte wirft die Frage auf, warum so viele Benutzer mit „weiß nicht” antworten. Grund hierfür mag die Tatsache sein, dass viele die Auskunft überhaupt nicht in Anspruch nehmen.

\section{Angebote}

Schließlich wurde den Benutzern die Frage gestellt, inwieweit ihnen die Angebote der Musiksammlung bekannt sind. Bei 93 Antworten war die Teilnahme an dieser Frage im Vergleich zu den anderen Fragen recht hoch.

Weniger als die Hälfte der Teilnehmenden hat Kenntnis von den entsprechenden Angeboten. Immerhin wissen 40 von 93 Personen Bescheid darüber, dass die WLB die Musikliteratur der württembergischen Verlage besitzt. In Anbetracht, dass die Aufbewahrung der württembergischen Pflichtexemplare eine der wesentlichen Aufgaben der Landesbibliothek ist, scheint diese Tatsache offensichtlich wenig bekannt zu sein. Damit zusammen hängt auch die Sammlung des Aufführungsmaterials der Staatstheater Stuttgart, welche immer wieder nicht mehr benötigtes Material an die Landesbibliothek abgeben. Es handelt sich hierbei zwar weniger um Pflichtexemplare, als vielmehr um einen Bestand mit starkem regionalen Bezug, der für die musikwissenschaftliche Forschung nicht unbedeutend ist. Dass nur 20 Personen von dem Angebot der Musikfachführungen wissen, überrascht wenig. Schließlich werden diese Führungen nur auf Anfrage angeboten, da sich die Nachfrage ohnehin in Grenzen hält. Diese Führungen bzw. Schulungen werden - wie oben bereits erwähnt in erster Linie im Rahmen von Semesterkursen für die HDMK angeboten. Auch die geringe Kenntnis über das Vorhandensein der Musikdatenbanken fällt auf: Bei nur 27 Personen ist das Wissen darüber vorhanden. Schließlich wissen auch nur 26 darüber Bescheid, dass ein Steinway-Flügel zum Anspielen von Noten zur Verfügung steht. 


\section{Kommentare, Wünsche, Kritik}

Wichtig für die gesamte Umfrage war das abschließend zur Verfügung gestellte Kommentarfeld, in welches Kommentare und Wünsche, aber auch Kritik und Verbesserungsvorschläge eingetragen werden durften. An dieser Stelle sollen die wichtigsten Aussagen aufgegriffen werden.

\section{Räumlichkeiten}

Nicht überraschend war die mehrfach geäußerte Kritik am Fehlen des bisher stets vorhandenen Musiklesesaals. Dass der Wegfall eines eigenen Fachlesesaals zunächst als Verlust gewertet wird, ist aus Nutzersicht verständlich. Dennoch sind die Vorteile hervorzuheben: Der Musiklesesaal musste von Montag bis Freitag von 10 bis 17 Uhr und am Samstag von 9 bis 13 Uhr besetzt werden. In der Regel wurden die täglichen sieben Stunden Präsenz von zwei Musikbibliothekarinnen übernommen, die regelmäßig Unterstützung durch die Leitung der Musiksammlung erhalten haben. Diese Aufsichtstätigkeit war einerseits sehr zeitintensiv und hat die Flexibilität der Kolleginnen erheblich eingeschränkt. Andererseits war dieses Konzept angesichts sinkender Benutzungszahlen ohnehin perspektivisch nicht mehr tragbar. Die Integration des Präsenzbestands in den Hauptlesesaal hat den entscheidenden Vorteil, dass dieser Bestand nun von 8 bis $20 \mathrm{Uhr}$ zugänglich ist, also fünf Stunden mehr pro Tag. Insofern ist dieser Präsenzbestand nun auch für berufstätige Benutzer besser verfügbar.

\section{Bestand}

Die Magazinierung verschiedener Gesamtausgaben und Monographien aufgrund mangelnden Platzes im Hauptlesesaal und allgemein schwacher Frequentierung des Bestands wurde lediglich einmal kritisiert. Völlig zu Recht wurde bemängelt, dass Literatur zu musikethnologischen und popularmusikwissenschaftlichen Fragestellungen vielfach nur per Fernleihe verfügbar ist. An dieser Stelle ist anzumerken, dass für Benutzer die Möglichkeit besteht, Anschaffungsvorschläge für gewünschte und nicht vorhandene Titel zu machen. Von dieser Option wird im Bereich der Literatur zur Musikwissenschaft aber eher selten Gebrauch gemacht.

\section{Kataloge}

Dass der Wunsch des Nachweises aller Literatur zur Musik, Noten und Tonträger in einem gemeinsamen Katalog besteht, ist leicht nachzuvollziehen. Die bisherige Recherche in primär zwei Katalogen im digitalisierten Zettelkatalog, in dem Notendrucke mit Erwerbung bis zum Jahr 1989 nachgewiesen sind und im WLB-Katalog (Online-Katalog) mit Erwerbung ab 1990 - verkomplizierte die Recherche erheblich. Im Zuge der Konversion des alten digitalisierten Zettelkatalogs wird sich dieser Zustand jedoch schon in Kürze ändern. Die Konversion durch eine externe Firma ist mittlerweile abgeschlossen, die Nachbearbeitungen stehen allerdings noch aus. Voraussichtlich zum Ende dieses Jahres werden alle Musikalien im WLB-Katalog online nachgewiesen sein, was die Recherche merkbar vereinfachen wird.

\section{Ausleihe}

Was in der Umfrage bereits klar zum Ausdruck kam, wird auch im Kommentarfeld nochmals deutlich: Es besteht der Wunsch nach Ausleihe der Musikmedien. An dieser Stelle soll angemerkt werden, dass sowohl in der Stadtbibliothek (Ebene Musik) als in der Bibliothek der HDMK die Notenausleihe nach Hause möglich ist.

\section{Auskunft}

Kritische Bemerkungen seitens der Nutzer hinsichtlich der Auskunftstätigkeit (was Musikalien betrifft) sind dadurch bedingt, dass die Trennung von Fachberatung und Aufsicht nicht allen ausreichend klar ist. Sicherlich besteht bei der Informationskompetenz seitens der nicht musikbibliothekarisch ausgebildeten Mitarbeiter weiterer Schulungsbedarf. Dennoch muss an dieser Stelle betont werden, dass der Schwerpunkt derjenigen, die im Sonderlesesaal am Auskunftsplatz sitzen, bei der Aufsicht, und nicht bei der Auskunft liegt. Besonders für detaillierte Anfragen zum Musikbestand ist es empfehlenswert, sich direkt mit den Musikbibliothekarinnen in Verbindung zu setzen.

\section{Praktische Konsequenzen (Fazit)}

Was für praktische Konsequenzen ergeben sich nun aus den Ergebnissen der Benutzerumfrage? Die Resultate bestätigen zu großen Teilen das, was bereits im Vorfeld vermutet wurde: Auch wenn 
eine grundsätzliche Zufriedenheit der Benutzer mit den Angeboten der Musiksammlung besteht, so gibt es doch in verschiedenen Bereichen Möglichkeiten, die Benutzungsbedingungen zu optimieren.

Ein wesentliches Ergebnis der Umfrage ist der Wunsch nach mehr Kommunikation der Angebote. An erster Stelle sei der Bedarf eines besseren Leitsystems genannt. Der Weg zum Sonderlesesaal ist allgemein nicht leicht zu finden, und die getrennte Benutzung der Musikbestände ist nicht unbedingt offensichtlich. Optimal wäre darüber hinaus ohnehin die Zusammenführung der Bestände an einen Ort. Da eine Änderung der Notenausleihe auf eine Ausleihe außer Haus zum jetzigen Zeitpunkt zu viel Zeit und Personal binden würde, wurde beschlossen, die Noten und Tonträger in Zukunft zunächst im Hauptlesesaal - statt wie bisher im Sonderlesesaal - auszugeben. Auf diese Weise werden die bisher örtlich getrennten Bestände der Musikalien (im Sonderlesesaal) und des Präsenzbestandes (im Hauptlesesaal) wieder zusammengeführt. Damit verbunden ist ein weiterer Vorteil: Der Bestand kann bei deutlich besseren Öffnungszeiten benutzt werden, nämlich von 8 bis 20 Uhr statt wie bisher von 10 bis 17 Uhr. $^{7}$ Dennoch wird zu einem späteren Zeitpunkt noch einmal die Frage der AußerHaus-Ausleihe von Noten zu diskutieren sein. Dabei spielt jedoch die Funktion der Landesbibliothek als Archivbibliothek für die württembergischen Pflichtexemplare eine nicht unerhebliche Rolle. Ob die Ausleihe von Tonträgern vor dem Hintergrund der immer größeren Bedeutung von Streamingdiensten künftig überhaupt noch von Relevanz ist, bleibt zunächst abzuwarten.

Dass bei der Frage nach den speziellen Angeboten der Musiksammlung ein hoher Anteil kaum oder keine Kenntnisse über die Angebote verfügt, erstaunt vor dem Hintergrund der Tatsache, dass diese Angebote über die Website kommuniziert werden. Es entsteht der Eindruck, dass die Hinweise auf der Website nicht wahrgenommen werden.
Es bleibt die Frage offen, welche Informationen auf der Website gelesen werden. Die Angebote der Musiksammlung müssten dort noch deutlicher zum Ausdruck kommen.

Was den Präsenzbestand zur Musikwissenschaft betrifft, so ist für die Zukunft im Rahmen der Sanierung und baulichen Veränderungen des Bestandsgebäudes eine Erweiterung des frei zugänglichen Bestands, vor allem der Gesamtausgaben, angedacht. Aufgrund des Bedarfs an mehr im Lesesaal aufgestellter Literatur zur Musikgeschichte BadenWürttembergs, soll auch dieser Bereich künftig vergrößert werden.

Ein weiterer Kritikpunkt ist die Website, die als Mittel zur Kommunikation fungiert. Die Katalogseite beinhaltete bisher zu viele Kataloge und Informationstexte. Dadurch wirkte sie unübersichtlich und war für die Recherche eher kontraproduktiv. Diese Seite wurde in Konsequenz bereits erheblich „verschlankt". Dabei wurden die umfangreichen inhaltlichen Informationen komprimiert. Zum Jahresende 2017 wird schließlich der digitalisierte Zettelkatalog (DigiKat bzw. Musik-AK) mit Abschluss der Nacharbeiten des Konversionsprojekts von der Website genommen werden. Alle Notenbestände der Musiksammlung sind dann vollständig im OPAC online recherchierbar.

Hinsichtlich der Erwerbung wird der Wunsch nach mehr Literatur im Bereich der Unterhaltungsmusik (Pop- und Rockmusik, Schlager, Musical etc.) künftig berücksichtigt und das Erwerbungsprofil entsprechend verändert werden.

Veränderungspotential besteht auch im Angebot von Führungen zum Musikbestand. Weil sich das Interesse alleine an den Beständen der Musiksammlung aber in Grenzen hält, wäre es denkbar, ein neues Führungskonzept im Hinblick auf alle Bestände der Sondersammlungen der WLB zu entwickeln.

Maria Gramlich 